INTERNATIONAL JOURNAL OF RESEARCHES IN BIOSCIENCES, AGRICULTURE AND TECHNOLOGY

(C) VISHWASHANTI MULTIPURPOSE SOCIETY (Global Peace Multipurpose Society) R. No. MH-659/13(N) www.vmsindia.org

\title{
EFFECT OF SOME FOOD PRESERVATIVES ON SEED MYCOFLORA AND PHYSIOLOGICAL PARAMETERS OF FENUGREEK
}

\author{
R. Gadewar ${ }^{1}$, S. Charjan ${ }^{1}$, P. Lambat ${ }^{1}$, A. Lambat ${ }^{1}$, \\ V. Babhulkar ${ }^{2}$, V. Kalamkar ${ }^{3}$ and P. N. Charde ${ }^{1}$ \\ ${ }^{1}$ Sevadal Mahila Mahavidyalaya and Research Academy, Nagpur \\ College of Agriculture, Nagpur \\ Mathuradas Mohata College, Nagpur
}

\begin{abstract}
The Four different preservatives, camphor, asafoetida, naphthalene and boric acid were used to test their efficacy in controlling the mycoflora of fenugreek. Boric acid followed naphthalene Camphor and asafetida eliminated majority of seed borne mycoflora of fenugreek. Maximum reduction in the germination, seedling length and seed vigour was observed by asafoetida followed by naphthalene and camphor. Boric acid showed higher germination, seedling length and seed vigour than the control (untreated).
\end{abstract}

\section{Introduction :}

Information on storage of seeds to preserve the viability and vigour from harvest to next planting season is of prime importance in any seed production and storage programme. In the present investigation efficacy of some locally available natural food preservatives was studied for protection of fenugreek (methi) seeds from mould infestation.

\section{Materials and Methods :}

One kg seeds of Fenugreek (Trigonella foenum-graecum L.) was taken in separate tins and $1 \mathrm{~g}$ of each compound (camphor, asafoetida, naphthalene and boric acid ) was placed and a lid was replaced. The seeds thus prepared were stored at room temperature $\left(27^{\circ} \mathrm{C}\right)$ for 30 days. At the ends of storage period the seed borne mycoflora germination, seedling length and seed vigour method (1) and dilution plate method (2) The food preservatives adversely affected seed mycoflora of fenugreek (Table 1) However, the effect varied with the preservative. Camphor was responsible for eliminating Aspergillus terreus, Drechshera halodes, Fusarium moniliforme, Mucor varians, Oedocephalum sp, Penicillium funiculosum, Alternaria alternate, Rhizoctonia solani and Verticillium cyclopium on the other hand, this encouraged the growth of $F$. heterosporum and A. niger. Napthalene inhibited the growth of Cladosporium cholorocephalum, D. halodes, M. lvarians, Oedocehalum sp, Paecilomyces varioti, $P$. aurantiogriseum and $R$. solani. Asofoetida (Hing) inhibited the growth of Alternaria, Aspergillus japonicas, $F$. oxalicum, $P$. funiculosum, $P$. verruculosum var. cyclopium, Humicola grisea, $R$. solani and Trichoderma koeningii. However, it promoted the growth of $C$. oxysporum, $F$. heterosporum, Phoma $s p$. Boric acid was found to be highly inhibitory to the growth of $A$. terricola, A. flavus, $P$. varioti, $P$. aurantigogriseum, $P$. oxalicum, $H$. grisea and $R$. solani. However, it was not effective to control the growth of Cladosporium oxysporum and $F$. heterosporum.

Maximum reduction in the germination, seedling length and seed vigour observed by asafoetida followed by naphthalene and camphor. Boric acid showed higher germination, seedling length and seed vigour than the control. Similarly Boric acid followed by naphthalene and camphor eliminated majority of seed borne mycoflora of fenugreek.

Table. 1. Effect of some food preservatives of seed mycoflora of fenugreek

\begin{tabular}{|l|l|l|l|l|l|l|l|l|l|l|}
\hline \multirow{2}{*}{ Name of the fungus } & \multicolumn{2}{|c|}{ Control } & \multicolumn{2}{c|}{ Camphor } & \multicolumn{2}{c|}{ Naphthalene } & \multicolumn{2}{c|}{ Asofoetida } & \multicolumn{2}{c|}{ Boric Acid } \\
\cline { 2 - 15 } & A & B & A & B & A & B & A & B & A & B \\
\hline Acremonium terricola & 2.4 & 7.5 & - & 10.4 & - & 1.2 & - & - & - & - \\
\hline Alternaria dianthicola & 4.0 & - & - & - & - & 1.0 & 1.4 & - & - & - \\
\hline Alternaria sp. & - & 1.0 & - & - & - & 1.0 & - & - & - & - \\
\hline Asergillus flavus & 18.2 & 1.2 & 1.2 & 1.2 & 3.5 & 1.2 & 10.0 & 10.2 & - & - \\
\hline A. Fumigatus & 10.4 & 10.1 & 2.6 & 3.5 & 10.2 & 10.6 & 6.8 & 7.4 & 8.4 & 10.2 \\
\hline A. Japonicas & 5.6 & 8.4 & 1.2 & 1.6 & 1.2 & 1.4 & 1.8 & - & 1.2 & 10.6 \\
\hline A. Niger & 30.2 & - & 7.6 & 2.6 & 15.2 & 1.0 & 20.0 & - & 3.6 & - \\
\hline A. Terrus & 1.2 & 8.1 & - & - & - & 2.6 & - & 6.2 & - & 2.4 \\
\hline Aspergillus sp. & - & 1.2 & - & 1.2 & - & 3.5 & - & 1.0 & - & - \\
\hline $\begin{array}{l}\text { Cladosporium } \\
\text { cholorocephalum }\end{array}$ & - & 2.2 & - & - & - & - & - & 1.0 & - & - \\
\hline C. cladosporioides & - & 2.6 & - & 5.4 & - & 10.2 & - & 10.2 & - & 5.2 \\
\hline
\end{tabular}




\begin{tabular}{|l|l|l|l|l|l|l|l|l|l|l|}
\hline C. oxysporum & 1.4 & - & - & 1.2 & - & - & - & 4.2 & - & 2.2 \\
\hline Curvularia lunata & - & - & - & - & - & 1.2 & - & - & - & - \\
\hline Drechslera halodes & - & 1.2 & - & - & - & - & - & 1.0 & - & - \\
\hline Epicoccum purpurescenes & 1.2 & 1.4 & - & - & - & - & - & - & - & - \\
\hline Fusarium heterosporum & 2.2 & - & 1.2 & 5.2 & - & 5.5 & 1.2 & 6.2 & - & 1.2 \\
\hline F. moniliforme & - & 1.2 & - & - & - & 3.6 & - & - & - & - \\
\hline Humicola grisea & - & 2.4 & - & 1.2 & - & 2.4 & - & - & - & - \\
\hline Mucor varians & 10.2 & 2.2 & 1.6 & - & 1.2 & - & 2.0 & - & 10.8 & 2.0 \\
\hline Oedocephalum sp. & - & 1.2 & - & - & - & - & - & - & - & - \\
\hline Paecilomyces varioti & - & 1.3 & - & 2.4 & - & - & - & 6.8 & - & - \\
\hline $\begin{array}{l}\text { Penicillium } \\
\text { aurantigogriseum }\end{array}$ & - & 1.2 & - & 1.3 & - & - & - & - & - & - \\
\hline P. Citrinum & 1.5 & 1.2 & - & 3.9 & 1.8 & 10.5 & 2.2 & 14.2 & - & 2.6 \\
\hline P. funiculosum & - & 2.6 & - & - & - & 3.6 & - & - & - & 1.8 \\
\hline P. oxalicum & 2.2 & 1.4 & - & 4.5 & - & 1.2 & - & - & - & - \\
\hline $\begin{array}{l}\text { P. verruculosum var. } \\
\text { cyclopium }\end{array}$ & - & 1.2 & - & 1.2 & - & 3.4 & - & - & - & 1.2 \\
\hline Phoma sp. & - & - & - & - & - & 1.2 & - & 1.8 & - & - \\
\hline Rhizoctonia solani & - & 1.2 & - & - & - & - & - & - & - & - \\
\hline $\begin{array}{l}\text { Syncephalastrum } \\
\text { recemosum }\end{array}$ & - & 1.4 & - & 1.4 & - & 1.0 & - & 1.0 & - & - \\
\hline Trichoderma koningii & - & 1.6 & - & 2.6 & - & 5.6 & - & - & - & 4.6 \\
\hline Verticillium cyclopium & 1.2 & 1.2 & - & - & - & 3.6 & - & 1.8 & - & 3.8 \\
\hline Sterile mycelia & 1.4 & - & - & 1.2 & - & - & - & 1.6 & - & 2.2 \\
\hline
\end{tabular}

$\mathrm{A}=$ Blotter technique, $\mathrm{B}=$ Dilution plate method.

Table 2. Effect of some food preservatives on germination and seedling vigour of fenugreek.

\begin{tabular}{|l|l|l|l|}
\hline Preservative & Germination \% & Seedling length $(\mathrm{cm})$ & Vigour index. \\
\hline Control & 91 & 11.42 & 1039.22 \\
\hline Camphor & 88 & 11.01 & 968.88 \\
\hline Naphthalene & 86 & 11.04 & 949.44 \\
\hline Asofoetida & 85 & 10.81 & 918.85 \\
\hline Boric acid & 94 & 12.46 & 1171.24 \\
\hline
\end{tabular}

\section{References:}

1. ANONYMOUS (1995) . International rules for seed testing Annexes, Seed Sci. \& Technol. 13: 356-513.
2. WAKSMAN, S.S. (1922) A method of counting the number of fungi in the soil. J. Bact. 7: 399-341. 